Y. Kato

Nagoya Math. J.

Vol. 128 (1992), 1-14

\title{
ON A BINGHAM FLUID WHOSE VISCOSITY AND YIELD LIMIT DEPEND ON THE TEMPERATURE
}

\author{
YOSHIO KATO
}

\section{Introduction}

Duvaut and Lions [2] studied the field of velocities and of temperatures in a moving incompressible Bingham fluid endowed with viscosity $\mu(\theta)$ depending on the temperature $\theta$ and established the existence of a weak solution in the case of a two dimensional fluid. However, the problem of uniqueness remained unsolved. The purpose of the present paper is to give an affirmative answer to the problem, that is, to show the local existence (resp. the global existence) in the time and the uniqueness of (strong) solutions in three dimensions under the conditions that (i) the time (resp. the initial velocity and the external force) and (ii) the rate of variation of the viscosity and the yield limit with respect to the temperature are both sufficiently small. It will be easily seen that the global existence and the uniqueness also hold in the two dimensional case whenever the rate (ii) is sufficiently small.

The general plan of the proof follows the analogous lines as in [2]. Let $\phi$ be a given function. We first find the unique velocity field $u_{\psi}$ of a Bingham fluid with viscosity $\mu(\phi)$ and yield limit $g(\phi)$, employing Theorem 3 of Kato [4], and secondly seek the solution $\theta_{\psi}$ of the heat equation $\theta_{t}-\Delta \theta=G_{\psi}$, the equation of energy-conservation associated with $u_{\psi}$, with the aid of the theorem due to Grisvard [3]. A desired field of temperature is obtained by a fixed point $\theta$ of the mapping $H: \psi \rightarrow \theta_{\psi}$ and $u_{\theta}$ is a desired field of velocity. The crucial point will be in finding an auxiliary Banach space $X$ to which $\phi$ belongs and on which mapping $H$ becomes compact, and in estimating the right hand side $G_{\psi}$ of the heat equation in terms of $\|\phi\|_{X}$ (see Lemma 2.2) so that a ball in $X$ is transformed into itself by mapping $H$ under some circumstances.

The main result, Theorem 1, is described in Section 1 . The aim of Section 2 is to get $u_{\psi}$ and $\theta_{\psi}$. Section 3 is devoted to the proof of Theorem 1 in which

Received July 22, 1991. 
Schauder's fixed point theorem will be applied to the mapping $H$.

\section{$\S 1$. Preliminaries and results obtained}

Throughout the paper we assume that $\Omega$ is a bounded domain in three dimensional euclidian space $\mathbf{R}^{3}$ with a smooth boundary $\Gamma$. For an integer $k \geq 0$ and $1 \leq p \leq \infty, W^{k, p}(\Omega)$ means the usual Sobolev space with norm $\|\cdot\|_{k, p}$. In particular, we set $W^{0, p}(\Omega)=L^{p}(\Omega)$ and $\|\cdot\|_{0, p}=\|\cdot\|_{p}$. Given an interval $I=[0, T]$ and a Banach space $B$, let us denote by $W^{k, p}(I ; B)$ the set of all $L^{p}$-functions $\phi$ of $I$ into $B$ such that

$$
\sum_{j=0}^{k} \int_{0}^{T}\left\|\phi^{(j)}(t)\right\|_{B}^{p} d t<\infty(p<\infty) \text { and } \sum_{j=0}^{k} \underset{t \in I}{\operatorname{ess} \sup }\left\|\phi^{(j)}(t)\right\|_{B}<\infty(p=\infty),
$$

where $\phi^{(0)}=\phi$ and $\phi^{(j)}, j \geq 1$, is the $j$-th order derivative with respect to $t$ in the distribution sense. It is well-known in the literature that if $\phi \in W^{1, p}(I ; B)$ for $p>1$, then we have $\phi \in C(I ; B)$, modifying, if necessary, the value of $\phi$ on a set of measure zero. Moreover, we can prove that for any $\varepsilon>0$ there exists a positive constant $C_{\varepsilon}$ such that

$$
\max _{t \in I}\|\phi(t)\|_{B} \leq \varepsilon\left(\int_{0}^{T}\left\|\phi^{\prime}\right\|_{B}^{p} d t\right)^{1 / p}+C_{\varepsilon}\left(\int_{0}^{T}\|\phi\|_{B}^{p} d t\right)^{1 / p} .
$$

We now introduce the function spaces:

$$
\begin{gathered}
V_{k, p}=\text { the closure of } \mathscr{V}(\Omega) \text { in } W^{k, p}(\Omega) \text { with norm }\|v\|_{V_{k, p}}=\|v\|_{k, p}, \\
\mathscr{H}_{P}=\left\{\phi \in L^{p}\left(I ; W^{2, p}(\Omega)\right) ; \phi^{\prime} \in L^{p}\left(I ; L^{p}(\Omega)\right)\right\} \text { with norm } \\
\|\phi\|_{\mathscr{H}_{p}}=\left(\int_{0}^{T}\left(\|\phi\|_{2, p}^{p}+\left\|\phi^{\prime}\right\|_{p}^{p}\right) d t\right)^{1 / p},
\end{gathered}
$$

and in particular we set

$$
V_{0,2}=H, \quad V_{1, p}=V_{p} \text { and } V_{2}=V,
$$

where $\mathscr{V}(\Omega)=\left\{\left(\varphi^{1}, \varphi^{2}, \varphi^{3}\right) ; \varphi^{i} \in C_{0}^{\infty}(\Omega)\right.$, div $\left.\varphi=0\right\}$. Identifying $H$ to its dual $H^{\prime}$, we obtain $V \subset H=H^{\prime} \subset V^{\prime}$, where $V^{\prime}$ is the dual space of $V$, each space is dense in the following and the injections are one to one and continuous. It is not difficult to see that

$$
\langle f, u\rangle=\int_{\Omega} f u d x \quad \text { for } f \in H \text { and } u \in V,
$$


where $\langle$,$\rangle denotes the duality between V$ and $V^{\prime}$.

Lemma 1.1. For any $p$ such that $1<p<2$ we set

$$
\frac{1}{\beta}=\frac{1}{p}-\frac{p-1}{3}
$$

Then, $1<\beta<6$ and $\mathscr{H}_{p} \subset L^{2}\left(I ; W^{1, \beta}(\Omega)\right)$. Moreover, for any $\varepsilon>0$ we can find a positive constant $K_{\varepsilon}$ so that

$$
\left(\int_{0}^{T}\|\phi\|_{1, \beta}^{2} d t\right)^{1 / 2} \leq \varepsilon\|\phi\|_{\mathscr{H}_{p}}+K_{\varepsilon}\left(\int_{0}^{T}\|\phi\|_{p}^{p} d t\right)^{1 / p}, \quad \phi \in \mathscr{H}_{p} .
$$

Proof. Observing the relation $p<\beta<p^{*}=3 p /(3-p)$, we have, by the interpolation inequality,

$$
\|\phi\|_{1, \beta} \leq \text { const. }\|\phi\|_{1, p}^{2-p}\|\phi\|_{1, p}^{p-1} .
$$

The use of Sobolev's inequality and the inequality

$$
\|\phi\|_{1, p}^{2} \leq \text { const. }\|\phi\|_{2, p}\|\phi\|_{p},
$$

which appears in $[1, \mathrm{p} .79]$, therefore implies

$$
\|\phi\|_{1, \beta}^{2} \leq \text { const. }\|\phi\|_{2, p}^{p}\|\phi\|_{p}^{2-p}, \quad \phi \in W^{2, p}(\Omega),
$$

from which (1.3) easily follows by using (1.1) with $B=L^{p}(\Omega)$ and Sobolev's imbedding theorem.

Q.E.D.

It is easily verified that $\sqrt{7}-1<p$ is equivalent to $p^{\prime}=p /(p-1)<\beta$, and $p \leq 5 / 3$ to $p^{\dagger}=2 p /(2-p) \leq 10$. From now on we fix $p$ so that

$$
\sqrt{7}-1<p \leq \frac{5}{3}
$$

and define $\alpha$ by

$$
\frac{1}{\alpha}+\frac{1}{\beta}=\frac{2}{3}
$$

Putting $p^{*}=3 p /(3-p)$, we then have, keeping in mind $1 / p^{\prime}+1 / p^{*}=2 / 3$,

$$
p^{\prime}<\beta<3<\alpha<p^{*}, \quad 6<p^{\dagger} \leq 10 \text { and } 6<\beta^{*} .
$$

If $a$ 's and $b$ 's are determined by 


$$
\frac{1}{a}+\frac{1}{b}=\frac{1}{2} \text { and } b=\beta^{*}
$$

it then immediately follows that

$$
\frac{1}{a}+\frac{1}{3}=\frac{1}{\alpha}+\frac{1}{2}
$$

The relation $\alpha<p^{*}$ implies that the injection of $W^{2, p}(\Omega)$ into $W^{1, \alpha}(\Omega)$ is compact. The reason why we claim $p^{\dagger} \leq 10$ will be found in the integral $\int_{0}^{T}\|D(u)\|_{p^{+}}^{\dagger} d t$ which appears in (2.13) to be finite it is necessary that $r \geq q$ in (2.4) and this occurs when and only when $q \leq 10$.

For $\alpha$ and $\beta$ from (1.2) and (1.5) we introduce the Banach space

$$
X=\left\{\phi \in C\left(I ; W^{1, \alpha}(\Omega)\right) ; \phi^{\prime} \in L^{2}\left(I ; W^{1, \beta}(\Omega)\right)\right\},
$$

equipped with norm

$$
\|\phi\|_{X}=\max _{t \in I}\|\phi(t)\|_{1, \alpha}+\left(\int_{0}^{T}\left\|\phi^{\prime}\right\|_{1, \beta}^{2} d t\right)^{1 / 2},
$$

which plays important roles in the paper. Let $Y_{0}$ amd $Y_{1}$ be two Banach spaces:

$$
Y_{0}=\left\{\phi \in \mathscr{H}_{p} ; \phi^{\prime} \in \mathscr{H}_{p}\right\} \quad \text { and } \quad Y_{1}=W^{1, p}\left(I ; L^{p}(\Omega)\right) \text {, }
$$

with respect norms,

$$
\|\phi\|_{Y_{0}}=\|\phi\|_{\mathscr{H}_{p}}+\left\|\psi^{\prime}\right\|_{\mathscr{H}_{p}} \text { and }\|\phi\|_{Y_{1}}=\left(\int_{0}^{T}\left(\|\phi\|_{p}^{p}+\left\|\phi^{\prime}\right\|_{p}^{p}\right) d t\right)^{1 / p} \text {. }
$$

Since (1.6) guarantees

$$
W^{2, p}(\Omega) \subset W^{1, \alpha}(\Omega) \subset W^{1, \beta}(\Omega) \subset L^{b}(\Omega) \subset L^{2}(\Omega),
$$

we readily obtain $X \subset Y_{1}$ with continuous injection. Furthermore, we can prove, by virtue of the relation $\alpha<p^{*}$,

Lemma 1.2. The space $Y_{0}$ is contained in $X$ in a manner that for any $\varepsilon>0$ there exists a positive constant $K_{\varepsilon}$ such that

$$
\|\phi\|_{X} \leq \varepsilon\|\phi\|_{Y_{0}}+K_{\varepsilon}\|\phi\|_{Y_{1}}, \quad \phi \in Y_{0} .
$$

Moreover, the injection $Y_{0} \rightarrow X$ is continuous and compact. 
Proof. As we have already seen, the compactness of the injection $W^{2, p}(\Omega) \rightarrow$ $W^{1, \alpha}(\Omega)$ follows from Sobolev's imbedding theorem. Therefore, the use of (1.1) with $B=W^{1, \alpha}(\Omega)$ yields that for any $\varepsilon>0$ there exists a positive constant $C_{\varepsilon}$ such that

$$
\max _{t \in I}\|\phi(t)\|_{1, \alpha} \leq \varepsilon \int_{0}^{T}\left(\left\|\phi^{\prime}\right\|_{2, p}+\|\phi\|_{2, p}\right) d t+C_{\varepsilon} \int_{0}^{T}\|\phi\|_{p}^{p} d t .
$$

The proof of (1.11) will be thus achieved by adapting (1.3) with $\phi=\psi^{\prime}$.

On account of the compactness of the injection $Y_{0} \rightarrow Y_{1}$ (for the proof see [5, p.58]), we can immediately conclude from (1.11) the later half of the lemma.

Q.E.D.

Let $\mu$ and $g$ be a viscosity coefficient and a yield limit, respectively, which are positive functions of $\lambda \in \mathbf{R}$ such that

$$
\mu, g \in C^{1}(\mathbf{R}), \quad \mu_{0} \leq \mu(\lambda) \leq \mu_{1} \quad \text { and } \quad g_{0} \leq g(\lambda) \leq g_{1}
$$

for some positive constants $\mu_{i}$ and $g_{i}(i=0,1)$. We can easily see that

$$
\mu(\phi) \in X \quad \text { and } \quad g(\phi) \in W^{1,2}\left(I ; L^{2}(\Omega)\right) \text { for } \phi \in X .
$$

More precisely, it follows from Sobolev's imbedding theorem that

$$
\text { (1.14) } \max _{t \in I}\|\nu \nabla \mu\|_{\alpha}+\left(\int_{0}^{T}\left\|\nu \mu_{t}\right\|_{b}^{2} d t\right)^{1 / 2}+\left(\int_{0}^{T}\left\|\sqrt{\nu} g_{t}\right\|^{2} d t\right)^{1 / 2} \leq c \omega\|\phi\|_{X},
$$

where $c$ is a positive constant, $\mu=\mu(\phi), g=g(\phi), \mu_{t}=\partial \mu / \partial t, g_{t}=\partial g / \partial t$, $\nu=1 / \mu$ and

$$
\omega=\omega(\mu, g)=\sup _{\lambda \in \mathbf{R}} \frac{\left|\mu^{\prime}(\lambda)\right|}{\mu(\lambda)}+\sup _{\lambda \in \mathbf{R}} \frac{\left|g^{\prime}(\lambda)\right|}{\sqrt{\mu(\lambda)}} .
$$

The problem we consider here is then formulated as follows. For prescribed data $f, u_{0}, \rho$ and $\theta_{0}$ :

$$
\begin{aligned}
f \in W^{1,1}(I ; H) \cap L^{\infty}\left(I ; L^{3}(\Omega)^{3}\right), \quad u_{0} \in V, \quad \rho \in Y_{1} \text { and } & \\
& \theta_{0} \in W^{2, p}(\Omega) \cap W_{0}^{1, p}(\Omega)
\end{aligned}
$$

find a pair $\{u, \theta\}$ :

$$
u \in L^{\infty}(I ; V), u^{\prime} \in L^{2}(I ; V) \cap L^{\infty}(I ; H), \quad \theta \in Y_{0}
$$

satisfying, the variational inequality corresponding to the equation of motion: 


$$
\begin{aligned}
\left\langle u^{\prime}(t)+B(u(t)), v-u(t)\right. & +\Phi(\theta(t), v) \\
& -\Phi(\theta(t), u(t)) \geq\langle f(t), v-u(t)\rangle
\end{aligned}
$$

for $v \in V$ and for a.e. $t \in I$, the equation of energy conservation:

$$
\theta^{\prime}-\Delta \theta+u \cdot \nabla \theta=F(\theta, u)+\rho \text { in } \Omega \times I
$$

and the initial-boundary conditions:

$$
\begin{aligned}
& u(0)=u_{0}, \quad \theta(0)=\theta_{0} \text { in } \Omega \\
& \theta=0 \text { on } \Gamma \times I,
\end{aligned}
$$

where $W_{0}^{1, p}(\Omega)=\left\{\theta \in W^{1, p}(\Omega) ; \theta=0\right.$ on $\left.\Gamma\right\}, B(u)=u \cdot \nabla u$,

$$
\Phi(\theta, u)=\int_{\Omega}\left(\mu(\theta)|D(u)|^{2}+g(\theta)|D(u)| d x\right.
$$

and

$$
F(\theta, u)=2 \mu(\theta)|D(u)|^{2}+g(\theta)|D(u)| .
$$

This problem will be resolved along the following line. For $\phi \in X$ such that $\phi(0)=\theta_{0}$ and $\phi=0$ on $\Gamma \times I$ we first seek a vector field $u=u_{\psi}$ satisfying $u(0)=u_{0}$ and (1.18) with $\theta$ replaced by $\phi$, and then find a solution $\theta_{\psi} \in Y_{0}$ of the heat equation $\theta^{\prime}-\Delta \theta=F\left(\phi, u_{\psi}\right)+\rho-u_{\phi} \cdot \nabla \phi$ subject to condition (1.20) (see Section 2). Secondly, we show that the mapping $H: \phi \rightarrow \theta_{\psi}$ of $X$ into $Y_{0}$ has a fixed point $\theta$ (see Section 3). It is evident that $\left\{u_{\theta}, \theta\right\}$ is a desired solution to the problem.

To do so we must impose on the initial data $\left\{u_{0}, \theta_{0}\right\}$ a condition; they are stationary solutions of (1.18)-(1.19), that is, they satisfy

$$
\left\langle B\left(u_{0}\right), v-u_{0}\right\rangle+\Phi\left(\theta_{0}, v\right)-\Phi\left(\theta_{0}, u_{0}\right) \geq\left\langle\chi, v-u_{0}\right\rangle, \quad v \in V
$$

for some $\chi \in H$ and

$$
\begin{aligned}
& -\Delta \theta_{0}+u_{0} \cdot \nabla \theta_{0}=F\left(\theta_{0}, u_{0}\right)+\rho(x, 0) \text { in } \Omega, \\
& \theta_{0}=0 \text { on } \Gamma .
\end{aligned}
$$

For the existence of such data we refer to Remark 3 in [4]. We now ready to state the main theorem.

Theorem 1. Suppose that $\Omega$ is a bounded domain in $\mathbf{R}^{3}$ with smooth boundary $\Gamma$, that $p$ satisfies (1.4) and that $\mu, g$ are functions on $\mathbf{R}$ which satisfy (1.13). Let $f$, $u_{0}, \rho$ and $\theta_{0}$ be given as in $(1,16),(1,23)$ and $(1,24)$. If at least one of two quantities 

(i) $\|\chi\|+\int_{0}^{T}\left(\|f\|+\left\|f^{\prime}\right\|\right) d t+\omega$ and
(ii) $T+\omega$

is sufficiently small, then there exists one and only one solution $\{u, \theta\}$ to the problem (1.18) (1.20) satisfying

$$
\begin{aligned}
& u \in L^{r}\left(I ; V_{q}\right) \quad \text { for all } r \leq 4 q /(q-6) \text { and all } 6 \leq q \leq 10 \\
& u^{\prime} \in L^{2}(I ; V) \cap L^{\infty}(I ; H) \text { and } \theta \in Y_{0} .
\end{aligned}
$$

Remark 1. Remembering [4, Remark 4], we can easily prove the following theorem. Suppose that $\Omega$ is a bounded domain in the plane with smooth boundary, that $p$ satisfies $\sqrt{5}-1<p<2$ and that $\mu, g$ are functions on $\mathbf{R}$ which satisfy (1.13). Let $f \in W^{1,1}(I ; H), u_{0} \in V, \rho \in Y_{1}$ and $\theta_{0} \in W^{2, p}(\Omega)$, and assume (1.23) and (1.24) to hold. If $\omega$ is sufficiently small, then there exists one and only one solution $\{u, \theta\}$ to the problem (1.18) (1.20) satisfying $u \in L^{\infty}\left(I ; V_{q}\right)$ for all $q<\infty, u^{\prime} \in$ $L^{2}(I ; V) \cap L^{\infty}(I ; H)$ and $\theta \in Y_{0}$.

\section{$\S 2$. The flow $u_{\psi}$ and the associated heat equation}

Throughout the section we assume the hypotheses mentioned in Theorem 1 to hold. Taking account of (1.5), (1.7) and (1.8), we can prove the following lemma by the same argument as in [4, Theorem 3].

Lemma 2.1. Let $\phi \in X$. If at least one of two conditions

$$
\text { (i) }\left(\mu_{0} / \gamma_{0}\right)^{4}>c_{0} A K_{2}^{2} \text { and (ii) } \mu_{0}^{2}>T^{1 / 2} K_{2}^{2}
$$

is fulfilled, then there exists exactly one vector field $u=u_{\psi}$ satisfying $u(0)=u_{0}$,

$$
\begin{aligned}
& \left\langle u^{\prime}(t)+B(u(t)), v-u(t)\right\rangle+\Phi(\phi(t), v) \\
& -\Phi(\phi(t), u(t)) \geq\langle f(t), v-u(t)\rangle, \\
& \left\|u^{\prime}(t)\right\|+\left(\frac{\mu_{0}}{4} \int_{0}^{T}\left\|\nabla u^{\prime}\right\|^{2} d t\right)^{1 / 2} \leq K_{1}
\end{aligned}
$$

and

$$
\begin{aligned}
& \|\nabla u(t)\|_{q} \leq K_{q} \text { when } 1<p \leq 6 \\
& \left(\int_{0}^{T}\|\nabla u\|_{q}^{r} d t\right)^{1 / r} \leq K_{q} \text { for } r=4 q /(q-6) \text { when } q>6
\end{aligned}
$$

for all $v \in V$ and for all $t \leq T$, where $\gamma_{0}, c_{0}$ are positive absolute constants, $\Phi$ the 
functional defined by (1.21),

$$
A=\left(\left\|u_{0}\right\|^{2}+\int_{0}^{T}\|f\| d t\right) \exp \left(\int_{0}^{T}\|f\| d t\right)
$$

and $K_{q}$ a positive continuous function of the arguments

$$
\mu_{0}, \mu_{1}, g_{1},\|\chi\|, \int_{0}^{T}\left(\|f\|+\left\|f^{\prime}\right\|\right) d t \text { and } \omega\|\phi\|_{X} .
$$

In particular,

$$
K_{1}+K_{2} \rightarrow 0 \quad \text { as }\|\chi\|+\int_{0}^{T}\left(\|f\|+\left\|f^{\prime}\right\|\right) d t+\omega \rightarrow 0,
$$

$\omega$ being defined by (1.15).

Proof. Following [4], we set

$$
\begin{gathered}
M=C \mu_{1} \mu_{0}^{-2}\left(\omega^{2}\|\phi\|_{X}^{2}+1\right) \omega^{2}\|\phi\|_{X}^{2}, \quad G=\omega^{2}\|\phi\|_{X}^{2}, \\
I=\left\{\|f(0)-\chi\|^{2}+\int_{0}^{T}\left\|f^{\prime}\right\| d t+\left(\max _{0 \leq t \leq T}\|f(t)\|^{2}+g_{1}^{2}\right) M+G\right\} \\
\times \exp \left(\int_{0}^{T}\left\|f^{\prime}\right\| d t+\gamma_{1} \mu_{0}+M\right), \\
J=M \exp \left(\int_{0}^{T}\left\|f^{\prime}\right\| d t+\gamma_{1} \mu_{0}+M\right)
\end{gathered}
$$

and

$$
E=\left(18 \mu_{0}^{\lambda-2} A^{1+\lambda} J\right)^{1 / \lambda}+18 \mu_{0} A J+\left\{18 A\left(\max _{0 \leq t \leq T}\|f(t)\|^{2}+I\right)\right\}^{1 / 2},
$$

where $\gamma_{1}$ and $C$ are some positive constants depending only on $\alpha$ and $\Omega$, and $\lambda=3 / \alpha-1 / 2<1 / 2$. Then, we obtain (2.4), provided

$$
\begin{aligned}
& K_{1}=2\left\{I+J\left(\mu_{0} E+\mu_{0}^{\lambda-2} A^{\lambda} E^{2-\lambda}\right\}^{1 / 2},\right. \\
& K_{q}=\text { const. } \mu_{0}^{-1 / 2} E^{1 / 2} \quad \text { when } 1<q \leq 2
\end{aligned}
$$

and

$$
K_{q}=\text { a positive function of argument (2.5) when } q>2 .
$$

Consequently, (2.6) is easily concluded.

For the further detail of the proof we refer to [4].

Q.E.D. 
We now treat the initial-boundary value problem, asscoiated with $\phi \in X_{0}$, for $\theta$ :

$$
\begin{aligned}
& \theta^{\prime}-\Delta \theta+u_{\psi} \cdot \nabla \phi=F\left(\phi, u_{\psi}\right)+\rho \text { in } Q=\Omega \times I, \\
& \theta-\theta_{0}=0 \text { on }(\Gamma \times I) \cup(\Omega \times\{0\}),
\end{aligned}
$$

where $I$ denotes the interval $[0, T], u_{\phi}$ the velocity field obtained in Lemma 2.1, $F(\phi, u)$ the same function as in (1.22) and

$$
X_{0}=\left\{\phi \in X ; \phi(0)=\theta_{0} \text { and } \phi=0 \text { on } \Gamma \times I\right\} .
$$

Setting $h=\theta-\theta_{0}$, we may rewrite (2.7) as

$$
\begin{aligned}
& h^{\prime}-\Delta h=\Delta \theta_{0}+F\left(\phi, u_{\psi}\right)+\rho-u_{\psi} \cdot \nabla \phi \text { in } Q, \\
& h=0 \text { on }(\Gamma \times I) \cup(\Omega \times\{0\}) .
\end{aligned}
$$

Let us set

$$
G_{\psi}=\Delta \theta_{0}+F\left(\phi, u_{\psi}\right)+\rho-u_{\psi} \cdot \nabla \phi \quad \text { for } \phi \in X_{0} .
$$

It is easily seen from (1.24) that the initial value of $G_{\psi}$ vanishes.

There is the key lemma in the present paper.

Lemma 2.2. Suppose that $\theta_{0}$ satisfies (1.24). Then, we have $G_{\psi} \in Y_{1}=$ $W^{1, p}\left(I ; L^{p}(\Omega)\right)$ for any $\phi \in X_{0}$. Moreover, the following estimate holds:

$$
\left\|G_{\psi}\right\|_{Y_{1}} \leq C_{T}\left\|\theta_{0}\right\|_{2, p}+M_{1}+C_{T}\left(K_{1}+K_{2}\right)\|\phi\|_{X},
$$

where $M_{1}$ is a positive continuous function of $T$ and the arguments (2.5), and $C_{T}$ is a positive constant depending only on $T$ such that $C_{T} \rightarrow 0$ as $T \rightarrow 0$.

Proof. Using Hölder inequality, we can derive from definition (1.22) the following inequalities:

$$
\begin{aligned}
& \|F\|_{p} \leq 2 \mu_{1}\|D(u)\|_{2 p}^{2}+g_{1}\|D(u)\|_{p}, \\
& \begin{aligned}
\left\|F^{\prime}\right\|_{p} \leq 2 \omega \mu_{1}\left\|\phi^{\prime}\right\|_{b}\|D(u)\|_{2 q}^{2}+4 \mu_{1}\left\|D\left(u^{\prime}\right)\right\|\|D(u)\|_{p^{+}} \\
+\omega \sqrt{\mu_{1}}\left\|\phi^{\prime}\right\|_{b}\|D(u)\|_{q}+g_{1}\left\|D\left(u^{\prime}\right)\right\|_{p},
\end{aligned}
\end{aligned}
$$

where $q=3 / p<2$ and $1 / p^{\dagger}=1 / p-1 / 2$. Keeping in mind $6<p^{\dagger} \leq 10$ (see (1.6)), $2 p<6$ and $2 q<6$, we can compute, using (2.3) and (2.4), as follows:

$$
\begin{aligned}
\left(\int_{0}^{T}\|F\|_{p}^{p} d t\right)^{1 / p} \leq & 2 \mu_{1}\left(\int_{0}^{T}\|D(u)\|_{2 p}^{2 p} d t\right)^{1 / p}+g_{1}\left(\int_{0}^{T}\|D(u)\|_{p}^{p} d t\right)^{1 / p} \\
& \leq\left(2 \mu_{1} K_{2 p}^{2}+g_{1} K_{p}\right) T^{1 / p}
\end{aligned}
$$


and

$$
\text { (2.13) } \begin{gathered}
\left(\int_{0}^{T}\left\|F^{\prime}\right\|_{p}^{p} d t\right)^{1 / p} \leq 2 \omega \mu_{1}\left(\int_{0}^{T}\left\|\phi^{\prime}\right\|_{b}^{2} d t\right)^{1 / 2}\left(\int_{0}^{T}\|D(u)\|_{2 q}^{2 p^{\dagger}} d t\right)^{1 / p^{\dagger}} \\
+4 \mu_{1}\left(\int_{0}^{T}\left\|D\left(u^{\prime}\right)\right\|^{2} d t\right)^{1 / 2}\left(\int_{0}^{T}\|D(u)\|_{p^{+}}^{p^{\dagger}} d t\right)^{1 / p^{\dagger}} \\
+\omega \sqrt{\mu_{1}}\left(\int_{0}^{T}\left\|\phi^{\prime}\right\|_{b}^{2} d t\right)^{1 / 2}\left(\int_{0}^{T}\|D(u)\|_{q}^{p^{\dagger}} d t\right)^{1 / p^{\dagger}}+g_{1}\left(\int_{0}^{T}\left\|D\left(u^{\prime}\right)\right\|_{p}^{p} d t\right)^{1 / p} \\
=\left(2 \mu_{1} K_{2 q}^{2}+\sqrt{\mu_{1}} K_{q}\right) T^{1 / p^{\dagger}} c \omega\|\phi\|_{X}+\text { const. }\left(\mu_{1} \mu_{0}^{-1 / 2} K_{p^{+}}+g_{1} T^{1 / p^{\dagger}}\right) K_{1} .
\end{gathered}
$$

In the same manner as above we get the following three estimates:

$$
\begin{gathered}
\left(\int_{0}^{T}\|u \cdot \nabla \phi\|_{p}^{p} d t\right)^{1 / p} \leq\left(\int_{0}^{T}\|u\|_{r}^{p} d t\right)^{1 / p} \sup \|\nabla \phi\|_{\alpha} \\
\leq \text { const. } T^{1 / p} K_{2}\|\phi\|_{X} \quad \text { with } 1 / r=1 / p-1 / \alpha>1 / 6, \\
\left(\int_{0}^{T}\left\|u \cdot \nabla \phi^{\prime}\right\|_{p}^{p} d t\right)^{1 / p} \leq\left(\int_{0}^{T}\left\|\nabla \psi^{\prime}\right\|_{\beta}^{2} d t\right)^{1 / 2}\left(\int_{0}^{T}\|u\|_{s}^{p^{\dagger}} d t\right)^{1 / p^{+}} \\
\leq \text {const. } T^{1 / p^{\dagger}} K_{2}\|\phi\|_{X} \quad \text { with } 1 / s=1 / p-1 / \beta>1 / 6
\end{gathered}
$$

and

$$
\begin{aligned}
& \left(\int_{0}^{T}\left\|u^{\prime} \cdot \nabla \phi\right\|_{p}^{p} d t\right)^{1 / p} \leq\left(\int_{0}^{T}\left\|u^{\prime}\right\|_{r}^{p} d t\right)^{1 / p} \sup \|\nabla \phi\|_{\alpha} \\
\leq & \text { const. } T^{1 / p^{\dagger}} K_{1}\|\phi\|_{X} \text { with } 1 / r=1 / p-1 / \alpha>1 / 6 .
\end{aligned}
$$

Thus, (2.11) easily follows from $(2.12) \sim(2.16)$.

Lemma 2.3. For any $\phi \in X_{0}$ there exists one and only one solution $h_{\psi} \in Y_{0}$ of the heat equation (2.9) satisfying

$$
\left\|h_{\psi}\right\|_{Y_{0}} \leq C_{1}\left\|G_{\psi}\right\|_{Y_{1}} .
$$

$C_{1}$ being a positive constant depending on $T, p$ and $Q=\Omega \times(0, T)$.

Proof. Let $h$ and $k$ be unique solutions contained in $\mathscr{H}_{p}$ of equations (2.9) and equation

$$
\begin{aligned}
& k^{\prime}-\Delta k=G^{\prime} \text { in } Q, \\
& k=0 \text { on }(\Gamma \times I) \cup(\Omega \times\{0\}),
\end{aligned}
$$

satisfying the inequalities

(2.19) $\|h\|_{\mathscr{H}_{p}} \leq C\left(\int_{0}^{T}\|G\|_{p}^{p} d t\right)^{1 / p}$ and $\|k\|_{\mathscr{H}_{p}} \leq C\left(\int_{0}^{T}\left\|G^{\prime}\right\|_{p}^{p} d s t\right)^{1 / p}$, 
respectively. The existence of such $h$ and $k$ is due to [3 Théorème 9.3]. Because of $G_{\psi}=0$ at $t=0$, we have $h^{\prime}=k$. Hence, it is easily seen that $h=h_{\psi}$ is in $Y_{0}$ and satisfies (2.17).

Q.E.D.

\section{§ 3. Proof of Theorem 1}

Lemmas 2.1, 2.2 and 2.3 enable us to introduce a mapping $H$ of $X_{0}$ into $Y_{0} \subset X$ (see (1.9), (1.10) and (2.8) for $X, Y_{0}$ and $X_{0}$, respectively):

$$
H: \phi \rightarrow \theta=H(\phi)=h_{\psi}+\theta_{0} .
$$

Regarding $H$ as a mapping of $X_{0}$ into itself, we can prove

Lemma 3.1. The mapping $H$ defined by (3.1) is continuous and compact on $X_{0}$.

Proof. The compactness of $H$ is an immediate consequence of Lemma 1.2. In fact, it is easily seen from (2.11) and (2.17) that $H(\phi)$ remains in a bounded set in $Y_{0}$ when $\|\phi\|_{X}$ is bounded.

We now prove the continuity of $H$. For $\psi$ and $\varphi$ belonging to $X_{0}$, we have, using abbreviations $\mu_{\psi}=\mu(\phi)$ and $g_{\phi}=g(\phi)$,

$$
\begin{aligned}
G_{\phi}-G_{\varphi} & =2\left(\mu_{\psi}-\mu_{\varphi}\right)\left|D\left(u_{\psi}\right)\right|^{2}+\left(g_{\psi}-g_{\varphi}\right)\left|D\left(u_{\psi}\right)\right| \\
& +2 \mu_{\varphi}\left\{\left|D\left(u_{\psi}\right)\right|+\left|D\left(u_{\varphi}\right)\right|+g_{\varphi}\right\}\left(\left|D\left(u_{\psi}\right)\right|-\left|D\left(u_{\varphi}\right)\right|\right) \\
& -\left(u_{\psi}-u_{\varphi}\right) \cdot \nabla \varphi-u_{\psi} \cdot \nabla(\phi-\varphi) .
\end{aligned}
$$

So that, setting $1 / r=1 / p-1 / 6$, we obtain, keeping in mind $r<3<\alpha<p$,

$$
\begin{aligned}
\left\|G_{\psi}-G_{\varphi}\right\|_{p} & \leq 2\left\|D\left(u_{\psi}\right)\right\|_{2 r}^{2}\left\|\mu_{\psi}-\mu_{\varphi}\right\|_{6}+\left\|D\left(u_{\psi}\right)\right\|_{r}\left\|g_{\psi}-g_{\varphi}\right\|_{6} \\
& +\mu_{1}\left(\left\|D\left(u_{\psi}\right)\right\|_{p^{+}}+\left\|D\left(u_{\varphi}\right)\right\|_{p^{\ddagger}}+\left\|g_{\varphi}\right\|_{p^{+}}\right\}\|\nabla z\| \\
& + \text { const. }\left(\|\nabla \varphi\|_{\alpha}\|\nabla z\|+\left\|\nabla u_{\psi}\right\|_{r}\|\nabla(\phi-\varphi)\|\right),
\end{aligned}
$$

and hence (2.4) leads to

$$
\left(\int_{0}^{T}\left\|G_{\psi}-G_{\varphi}\right\|_{p}^{p} d t\right)^{1 / p} \leq c_{1}\left(\int_{0}^{T}\|\nabla(\phi-\varphi)\|^{2} d t\right)^{1 / 2}+c_{2}\left(\int_{0}^{T}\|\nabla z\|^{2} d t\right)^{1 / 2},
$$

because $p^{\dagger}$ does not exceed 10 . Here, $z=u_{\psi}-u_{\varphi}$ and $c_{i}(i=1,2, \ldots)$ denote positive constants which depend on $\varphi$ and $\phi$ but remain bounded as far as they run over a bounded set of $X$.

On the other hand, by the usual argument it follows from (2.2) that

$$
\frac{1}{2} \frac{d}{d t}\|z\|^{2}+\mu_{0}\|\nabla z\|^{2} \leq\left\langle B(z), u_{\psi}\right\rangle-2\left\langle\left(\mu_{\psi}-\mu_{\varphi}\right) D\left(u_{\psi}\right), D(z)\right\rangle
$$




$$
\begin{gathered}
-\int_{\Omega}\left(g_{\varphi}-g_{\varphi}\right)\left(\left|D\left(u_{\psi}\right)\right|-\left|D\left(u_{\varphi}\right)\right|\right) d x \\
\leq \gamma\|z\|^{1 / 2}\left\|\nabla u_{\psi}\right\|\|\nabla z\|^{3 / 2}+2\left\|\mu_{\varphi}-\mu_{\varphi}\right\|_{p^{\prime}}\left\|D\left(u_{\psi}\right)\right\|_{p^{+}}\|D(z)\| \\
+\left\|g_{\varphi}-g_{\varphi}\right\|\|D(z)\| \\
\left.\leq \frac{1}{2} \mu_{0}\|\nabla z\|^{2}+\frac{c}{2}\left(\|z\|^{2}+\left\|D\left(u_{\psi}\right)\right\|_{p^{+}}^{2} \| \mu_{\psi}-\mu_{\varphi}\right)\left\|_{p^{\prime}}^{2}+\right\| g_{\varphi}-g_{\varphi} \|^{2}\right),
\end{gathered}
$$

where we used the relation $1 / p^{\prime}+1 / p^{\dagger}=1 / 2$. Therefore, we have

$$
\left(e^{-c t}\|z(t)\|^{2}\right)^{\prime}+\mu_{0} e^{-c t}\|\nabla z\|^{2} \leq c_{3}\left(\left\|D\left(u_{\psi}\right)\right\|_{P^{\dagger}}^{2}\left\|\mu_{\psi}-\mu_{\varphi}\right\|_{p^{\prime}}^{2}+\left\|g_{\psi}-g_{\varphi}\right\|^{2}\right) .
$$

Integrating the both sides of the above from $t=0$ to $t=T$, we obtain

$$
\|z(t)\|+\left(\int_{0}^{T}\|\nabla z\|^{2} d t\right)^{1 / 2} \leq c\left(\int_{0}^{T}\|\phi-\varphi\|_{p^{\prime}}^{p^{\prime}} d t\right)^{1 / p^{\prime}} .
$$

Hence,

$$
\left.\left(\int_{0}^{T}\left\|G_{\psi}-G_{\varphi}\right\|_{p}^{p} d t\right)^{1 / p} \leq c_{4} \| \phi-\varphi\right) \|_{X}
$$

We now return to the proof of the continuity of $H$. Let $\left\{\phi_{n}\right\}$ be a sequence in $X_{0}$ such that $\phi_{n} \rightarrow \phi$ in $X$ and set $h_{n}=h_{\psi_{n}}$ and $G_{n}=G_{\psi_{n}}$. Since the mapping $H$ is compact, we can find a subsequence $\left\{h_{m}\right\}$ of $\left\{h_{n}\right\}$ so that $h_{m} \rightarrow \tilde{h}$ in $X$. On the other hand from (2.19) and (3.3) it follows that there exists a positive constant $C$ such that

$$
\left\|h_{\phi}-h_{n}\right\|_{\mathscr{H}_{p}} \leq C\left\|\phi-\phi_{n}\right\|_{X} .
$$

Consequently, we have $\tilde{h}=h_{\psi}$, which implies $h_{n} \rightarrow h_{\psi}$ in $X$ as $n \rightarrow \infty$.

Q.E.D.

Lemma 3.2. There exists a positive number $R$ such that the ball in $X_{0}: B_{R}$ $=\left\{\phi \in X_{0} ;\left\|\phi-\theta_{0}\right\|_{X} \leq R\right\}$ is transformed into itself by the mapping $H$, if either quantity (i) or (ii) of (1.25) is sufficiently small.

Proof. Linking (2.11) with (2.17), we get

$$
\begin{array}{r}
(3.4)\left\|\theta-\theta_{0}\right\|_{X}=\left\|h_{\psi}\right\|_{X} \leq C_{0}\left\|h_{\psi}\right\|_{Y_{0}} \leq \frac{R}{2}+C_{0} C_{1} C_{T}\left(K_{1}+K_{2}\right)\left(R+\left\|\theta_{0}\right\|_{X}\right) \\
\text { for } \phi \in B_{R},
\end{array}
$$

where $C_{0}$ is a positive constant depending only on $T, p$ and $Q$, and $R$ is chosen as

$$
\frac{R}{2} \geq C_{0} C_{1} \sup \left(C_{T}\left\|\theta_{0}\right\|_{2, p}+M_{1}+\left\|\theta_{0}\right\|_{X}\right)
$$


where the supremum is taken over all arguments such that

$$
T+\|\chi\|+\int_{0}^{T}\left(\|f\|+\left\|f^{\prime}\right\|\right) d t+\omega\|\phi\|_{X} \leq K
$$

for some $K$. If the quantity (i) of (1.25) (resp., (ii) of (1.25)) is so small that $\omega<K / R$, and two inequalities (i) of (2.1) (resp. (ii) of (2.1)) and

$$
C_{0} C_{1} C_{T}\left(K_{1}+K_{2}\right) \leq \frac{1}{3}
$$

hold true, it then follows from (3.4) that $H(\phi) \in B_{R}$ (cf. (2.6) and (2.11)). Q.E.D.

Since $B_{R}$ is a closed convex and bounded subset of $X$, Lemmas 3.1 and 3.2 allow us to adapt the Schauder fixed point theorem to get a fixed point $\theta: H(\theta)$ $=\theta$. It is easily seen that $\left\{u_{\theta}, \theta\right\}$ is a solution to the problem $(1.18) \sim(1.20)$ satisfying (1.26).

Our final goal is to establish the uniqueness of $\left\{u_{\theta}, \theta\right\}$. Let us suppose $\left\{u_{\psi}, \phi\right\}$ be another solution. Then, we have $\eta^{\prime}-\Delta \eta=G_{\theta}-G_{\psi}$, where $\eta=\theta$ $-\phi$. Multiplying the both sides by $\eta$ and integrating on $\Omega$, we get, using hölder's inequality,

$$
\begin{gathered}
\frac{1}{2} \frac{d}{d t}\|\eta\|^{2}+\|\nabla \eta\|^{2}=\int_{\Omega} \eta\left(G_{\theta}-G_{\psi}\right) d x \\
\leq C \int_{\Omega}\left\{\eta^{2}\left(\left|D\left(u_{\psi}\right)\right|^{2}+\left|D\left(u_{\phi}\right)\right|\right)+|\eta||D(z)|\left(\left|D\left(u_{\psi}\right)\right|+\left|D\left(u_{\theta}\right)\right|+g_{\theta}\right)\right. \\
+|\eta||z||\nabla \theta|\} d x \\
\leq C\left\{\|\eta\|_{p^{\prime}}^{2}\left(\left\|D\left(u_{\psi}\right)\right\|_{p^{+}}^{2}+\left\|D\left(u_{\psi}\right)\right\|_{p^{+} / 2}\right)\right. \\
\left.+\|\eta\|_{p^{\prime}}\|\nabla z\|\left(\left\|D\left(u_{\phi}\right)\right\|_{p^{+}}+\left\|D\left(u_{\theta}\right)\right\|_{p^{+}}+\left\|g_{\theta}\right\|_{p^{+}}\right)+\|\eta\|_{p^{\prime}}\|z\|\|\nabla \theta\|_{\alpha}\right\},
\end{gathered}
$$

where and in the following we denote by $C$ various positive constants and $z=$ $u_{\theta}-u_{\psi}$. Integration from 0 to $t$ yields by (3.2)

$$
\begin{gathered}
\frac{1}{2}\|\eta(t)\|^{2}+\int_{0}^{t}\|\nabla \eta\|^{2} d \tau \leq C\left(\int_{0}^{t}\|\eta\|_{p^{\prime}}^{p^{\prime}} d \tau\right)^{2 / p^{\prime}} \\
+C\left(\int_{0}^{t}\|\eta\|_{p^{\prime}}^{\prime} d \tau\right)^{1 / p^{\prime}}\left\{\left(\int\|\nabla z\|^{2} d \tau\right)^{1 / 2}+\max _{0 \leq \tau \leq t}\|z(\tau)\|\right\} \leq C\left(\int_{0}^{t}\|\eta\|_{p^{\prime}}^{p^{\prime}} d \tau\right)^{2 / p^{\prime}}
\end{gathered}
$$

which implies

$$
\left.\|\eta(t)\|^{p^{\prime}}+\int_{0}^{t}\|\nabla \eta\|^{2} d \tau\right)^{p^{\prime} / 2} \leq C \int_{0}^{t}\|\eta\|_{p^{\prime}}^{p^{\prime}} d \tau .
$$

Observing $2<p^{\prime}<6$, we obtain by virtue of the interpolation inequality

$$
\text { LHS of }(3.5) \leq C \int_{0}^{t}\|\eta\|^{\alpha p^{\prime}}\|\eta\|_{6}^{\beta p^{\prime}} d \tau,
$$


where $\alpha=\left(6-p^{\prime}\right) / 2 p^{\prime}, \beta=3\left(p^{\prime}-2\right) / 2 p^{\prime}$ and hence $\beta p^{\prime}<2$. Setting

$$
k=2 \alpha p^{\prime} /\left(2-\beta p^{\prime}\right)=2\left(6-p^{\prime}\right) /\left(10-3 p^{\prime}\right),
$$

we have by Hölder's, Young's and Sobolev's inequality

$$
\begin{gathered}
\text { LHS of }(3.5) \leq C\left(\int_{0}^{t}\|\nabla \eta\|^{2} d \tau\right)^{\beta p^{\prime} / 2}\left(\int_{0}^{t}\|\eta\|^{k} d \tau\right)^{\left(2-\beta p^{\prime}\right) / 2} \\
\quad \leq\left(\int_{0}^{t}\|\nabla \eta\|^{2} d \tau\right)^{p^{\prime} / 2}+C\left(\int_{0}^{t}\|\eta\|^{k} d \tau\right)^{\left(2-\beta p^{\prime}\right) / 2 \alpha}
\end{gathered}
$$

and hence

$$
\|\eta(t)\|^{k} \leq C \int_{0}^{t}\|\eta\|^{k} d \tau,
$$

which immediately implies $\eta=0$ and $\left\{u_{0}, \theta\right\}=\left\{u_{\psi}, \phi\right\}$.

Q.E.D.

The following remark is suggested by Prof. Yoshio Tsutsumi.

Remark. It is evident that the sequence $\theta_{n}=H^{n}\left(\theta_{0}\right)(n=0,1,2, \ldots)$ is contained in $B_{R} \cap Y_{0}$ and satisfies $\left\|\theta_{n}-\theta_{0}\right\|_{Y_{0}} \leq R / C_{0}$. Making use of Lemmas 1.2 and 3.1 , we can extract a subsequence $\left\{\theta_{n^{\prime}}\right\}$ which converges to a fixed point $\theta$ in $X$. The uniqueness of the fixed point therefore implies $\left\|\theta_{n}-\theta\right\|_{X} \rightarrow 0$ as $n \rightarrow \infty$. This fact is suggestive of applying the contraction mapping theorem, in other words, establishing the inequality $\left\|G_{\varphi}-G_{\phi}\right\|_{Y_{1}} \leq \varepsilon\|\varphi-\phi\|_{X}$ for sifficiently small $\varepsilon>0$. However, it seems impossible to the author, because of the term $g_{1}(\phi)\left|D\left(u_{\psi}\right)\right|$.

\section{REFERENCES}

[1] R. A. Adams, Sobolev spaces, Academic Press, 1975.

[2] G. Duvaut and J. L. Lions, Transfert de chaleur dans un fluide de Bingham dont la viscosité dépend de la température, J. Funct. Anal., 11 (1972), 93-110.

[3] P. Grisvard, Équation différentielles abstraites, Ann. Sci. École Norm. Sup., (4) 2 (1969), 311-395.

[4] Y. Kato, Variational inequalities of Bingham type in three dimensions, to appear in Nagoya Math. J.

[5] J. L. Lions, Quelque méthodes de resolution des problémes aux limites non linéaires, Dunod/Gauthier-Villars, Paris, 1969.

Department of Applied Physics

School of Engineering

Nagoya University, Nagoya 464-01 JAPAN 